\title{
Tracionamento de caninos inclusos: diagnóstico e terapêutica
}

\author{
Impacted canine traction: diagnosis and treatment \\ Tracción de caninos incluidos: diagnóstico y tratamiento \\ Simone Carrijo DAMANTE ${ }^{1}$ \\ Weder Carneiro LOPES ${ }^{2}$ \\ Cesar Diogo Benichio RODRIGUES ${ }^{3}$ \\ Manuel Martin ADRIAZOLA ${ }^{4}$ \\ André Pinheiro de Magalhães BERTOZ ${ }^{5}$ \\ Renato BIGLIAZZI ${ }^{5}$ \\ ${ }^{1}$ Cirurgiã-Dentista pela Faculdade de Odontologia de Araçatuba Univ Estadual Paulista - UNESP, \\ 16015-050 Araçatuba - SP, Brasil \\ ${ }^{2}$ Cirurgião-Dentista pela Univ Estadual Paulista - UNESP, Mestrando em Implantodontia, Faculdade São Leopoldo Mandic, \\ 13045-755 Campinas-SP, Brasil \\ ${ }^{3}$ Graduando em Odontologia, Faculdade de Odontologia de Araçatuba Univ Estadual Paulista - UNESP, \\ 16015-050 Araçatuba - SP, Brasil \\ ${ }^{4}$ Mestrando, Programa de Pós-Graduação em Odontologia, Área de Concentração Ortodontia, \\ Faculdade de Odontologia de Araçatuba, Univ Estadual Paulista - UNESP, 16015-050 Araçatuba - SP, Brasil \\ ${ }^{5}$ Professor Assistente Doutor, Departamento de Odontologia Infantil e Social, Faculdade de Odontologia de Araçatuba \\ Univ Estadual Paulista - UNESP, 16015-050 Araçatuba - SP, Brasil
}

\section{Resumo}

Os caninos permanentes podem sofrer alterações no período de transição da dentadura mista para permanente devido a inúmeros fatores, sendo alguns deles a complexidade da trajetória de erupção, condições patológicas (odontomas, cistos e dentes supranumerários), ausência de espaço para erupção no arco dentário e trauma dos dentes decíduos. Depois dos terceiros molares o canino é o elemento que mais sofre impacção, sendo mais comum no gênero feminino e mais comum na região palatina. As impacções dentárias podem ocasionar distúrbios no desenvolvimento da oclusão e comprometimento dos dentes e estruturas adjacentes, todavia quanto mais precoce o diagnóstico melhor será para realizar o tratamento. O presente trabalho tem como objetivo analisar na literatura os métodos existentes de tracionamento de caninos impactados visando o melhor diagnóstico e terapêutica. O sucesso no tratamento depende do planejamento adequado de cada caso, para isso é necessário exames clínicos, radiografias e ou tomografias que fornecerão a localização precisa do elemento impactado. A técnica de tracionamento utilizada poderá ser escolhida de acordo com a habilidade de cada profissional, sendo que a intervenção ortodôntica terá resultados mais favoráveis em casos descobertos precocemente, em indivíduos de menor idade, com espaço presente no arco dentário e ausências de dilacerações apicais. Descritores: Dente Não Erupcionado; Dente Impactado; Dente Canino.

\section{Abstract}

Permanent canines may to suffer changes in the transition period from mixed to permanent dentures due to innumerable factors, some of them being the complexity of the eruption trajectory, pathological conditions (Odontomas, cysts and supernumerary teeth), absence of space for eruption in the dental arch and trauma of deciduous teeth. After the third molars the canine is the most impacted element, being more common in the female genus and more common in the palatal region. Dental impactions can cause disturbances in the development of occlusion and impairment of adjacent teeth and structures, however the earlier the better the diagnosis will be to perform the treatment. The present work aims to analyze in the literature the existing methods of traction of impacted canines aiming at the best diagnosis and therapeutics. Success in treatment depends on the proper planning of each case, for which clinical examinations, $x$-rays and or tomographies are necessary that will provide the precise location of the impacted element. The technique of traction used may be chosen according to the skill of each professional, and the orthodontic intervention will have more favorable results in cases discovered early in individuals of lesser age, with space present in the dental arch and absences of apical dilations. Descriptors: Tooth, Unerupted; Tooth, Impacted; Cuspid.

\section{Resumen}

Los caninos permanentes pueden sufrir alteraciones en el período de transición de la dentadura mixta a permanente debido a innumerables factores, siendo algunos de ellos la complejidad de la trayectoria de erupción, condiciones patológicas (odontomas, quistes y dientes supranumerarios), ausencia de espacio para erupción en el arco dental y, El trauma de los dientes decimales. Después de los terceros molares el canino es el elemento que más sufre impactación, siendo más común en el género femenino y más común en la región palatina. Las repercusiones dentales pueden ocasionar disturbios en el desarrollo de la oclusión y el compromiso de los dientes y estructuras adyacentes, sin embargo cuanto más precoz es el diagnóstico mejor será para realizar el tratamiento. El presente trabajo tiene como objetivo analizar en la literatura los métodos existentes de tracción de caninos impactados para el mejor diagnóstico y terapéutica. El éxito en el tratamiento depende de la planificación adecuada de cada caso, para ello es necesario exámenes clínicos, radiografías y / o tomografías que proporcionarán la localización precisa del elemento impactado. La técnica de tracción utilizada podrá ser elegida de acuerdo con la habilidad de cada profesional, siendo que la intervención ortodóntica tendrá resultados más favorables en casos descubiertos precozmente, en individuos de menor edad, con espacio presente en el arco dental y ausencias de dilataciones apicales.

Descriptores: Diente no Erupcionado; Diente Impactado; Diente Canino.

\section{INTRODUÇÃO}

Os caninos são dentes de extrema importância para proteção do sistema estomatognático, participando da função e harmonia oclusal e estética, sendo indispensáveis nos movimentos de lateralidade ${ }^{1}$.

Considerando a função desse elemento no arco dentário e na relação oclusal, a abordagem desse estudo de caninos inclusos tem grande relevância na Ortodontia, a fim de diagnosticar, intervindo precocemente para reduzir, ou evitar possíveis complicações tardias dessa anomalia ${ }^{2}$.

O canino permanente leva duas vezes mais tempo para completar sua erupção quando comparado com os demais elementos dentários, devido ao seu longo e complexo caminho de erupção, o que o torna mais susceptível a sofrer alteração na trajetória de erupção desde a odontogênese até o estabelecimento da oclusão normal, resultando em erupção ou impactação ${ }^{3}$.

O diagnóstico da impactação é realizado pela anamnese, exame clínico e radiográfico. $\mathrm{Na}$ análise do dente impactado é importante observar a idade do paciente e seus antecedentes familiares de agenesia ou retenções dentárias, sendo que o prognóstico do tratamento depende da posição do canino em relação aos dentes adjacentes e sua altura no 
processo alveolar ${ }^{4}$

A literatura aponta como causas locais de impactação os seguintes fatos: trajeto de irrupção longo e tortuoso, pois é um dos últimos dentes a irromper na cavidade bucal; falta de espaço no arco dentário; distúrbios na sequência de irrupção dos dentes permanentes; trauma dos dentes decíduos; agenesia dos incisivos laterais permanentes; máposição do germe dentário; dilaceração radicular e a anquilose dos caninos permanentes; retenção prolongada ou perda prematura do canino decíduo predecessor e presença de cistos; tumores ou supranumerários na região - servindo como obstáculo e a fissura alveolar ${ }^{5,6}$.

A prevalência de retenção ocorre nas seguintes condições: de $0,9 \%$ a $2,5 \%$ com maior frequência unilateral; de $75 \%$ a $95 \%$ dos casos no gênero feminino duas a três vezes mais que no gênero masculino; de 60 a $80 \%$ dos casos estão localizados por palatino ${ }^{7}$, sendo que a impacção de caninos é 10 vezes maior na maxila do que na mandíbula ${ }^{8,9}$.

As impacções podem ocasionar problemas como reabsorção das raízes dos dentes vizinhos, perda do comprimento do arco dentário, formação de cisto dentígero, infecções locais e dor reflexa ${ }^{4}$. No tratamento dessa anomalia existem suas vantagens e riscos; como a anquilose, a perda de vitalidade do dente, as reabsorções do canino e dentes adjacentes, perda do tecido de sustentação, recessão gengival e formação de bolsa periodontal, além do tempo de tratamento estendido ${ }^{10}$.

As implicações caso o canino impactado não seja tracionado podem ser agravadas uma vez que ele estará alojado em local não apropriado, sendo elas: a migração de dentes vizinhos, a reabsorção de suas raízes, má posição do dente impactado, reabsorções internas, a formação de cistos dentígeros, infecção relacionada com erupção parcial, em alguns casos dor referida devido à compressão de algum feixe vásculo nervoso, no entanto existe controversa e a impactação passar sem efeitos durante toda a vida do paciente $^{11}$. O propósito do presente trabalho foi, com base em revisão de literatura, analisar os métodos existentes de tracionamento de caninos impactados visando o melhor diagnóstico e terapêutica.

\section{REVISÃO DA LITERATURA}

As sucessões de desvios na sequência normal do desenvolvimento da oclusão podem levar as impactações dentárias. Além disso, dentes impactados podem causar agravantes como reabsorção das raízes dos dentes vizinhos, perda do comprimento do arco, formação de cisto dentígero, infecções locais, dor reflexa etc ${ }^{12}$. Depois dos terceiros molares, os caninos superiores permanentes têm a maior incidência de impactação ${ }^{13-15}$ manifestando-se estatisticamente em $2 \%$ da população. Sua incidência é aproximadamente duas vezes maior no sexo feminino podendo ocorrer de forma uni ou bilateral. Aproximadamente, $80 \%$ a $90 \%$ dos caninos impactados estão por palatino e $10 \%$ a $20 \%$ por vestibular ${ }^{13}$.

Em um estudo sobre a relação da prevalência e posição de caninos inclusos em relação à reabsorção radicular foi encontrada prevalência de 3,5\% de impacção em determinada população estudada, com maioria dos elementos em posição mesioangulada, mostrando-se uma prevalência maior que a apontada em dados anteriores e, portanto não se deve excluir a presença de viés por amostragem. Além disso, também houve neste estudo $15,4 \%$ dos casos de caninos retidos com determinado grau de reabsorção exclusivamente no gênero feminino e na faixa etária dos 11 aos 30 anos. Convergindo com os dados descritos anteriormente, esse estudo mostra que a idade foi um fator que estaticamente influenciou na presença de caninos retidos, acometendo mais faixas etárias de 13 a 30 anos, e quanto à reabsorção também evidencia a maior prevalência nessa mesma faixa etária concordando com a literatura e apresentando baixa incidência de reabsorção de inciso lateral ${ }^{16}$.

Com intuito de melhor planejamento alguns autores avaliaram o uso de tomografia computadorizada para diagnóstico de caninos inclusos, visto que é um exame com imagem tridimensional, em contrapartida com a radiografia que fornece apenas imagem bidimensional ${ }^{17}$, essas reconstruções multiplanares fornecem a exata localização do dente. Além disso, fornece a distancia correta das estruturas adjacentes, condições patológicas existentes e auxiliam no planejamento do tratamento e prognóstico da evolução do caso. Esse estudo analisou algumas tomografias, com o canino que se apresentava por vestibular em intima relação com incisivos, outra se apresentando por palatino sem intimidade com os incisivos e ainda outra situação promovendo reabsorção radicular externa dos dentes adjacentes, sendo essa ultima a que mais preocupa os profissionais. As condutas de tratamento do canino irão depender de cada caso e estruturas associadas, e caso o paciente não deseje tratar deve-se fazer o acompanhamento do caso para controle. Em se tratando de caninos inclusos é necessário o exato diagnóstico assim a associação da tomografia computadorizada é de fundamental importância para se analisar com rigorosidade a localização, posição e a relação com estruturas e dentes adjacentes, para que assim seja estabelecido um tratamento adequado para o caso ${ }^{18}$.

Em um estudo realizado para avaliação dos diferentes posicionamentos de caninos superiores não irrompidos em radiografias panorâmicas foram analisadas 4.350 radiografias panorâmicas, das quais, selecionaram-se setenta. Apenas um examinador realizou os traçados manualmente, sendo vinte deles repetidos posteriormente para obtenção do erro do método ${ }^{19}$.

Foram utilizados alguns métodos de localização: orientação horizontal, vertical, angulação, distancia da cúspide do canino ao plano oclusal e a crista óssea alveolar. Considerando-se as 70 radiografias analisadas, obtiveram $5,71 \%$ do gênero feminino e $34,29 \%$ do masculino. As impacções manifestaram-se com 55 casos unilaterais, sendo 31 no lado direito e 24 no lado esquerdo e 15 casos de impacção bilateral. Como visto por outros autores Ericsson e Kurol ${ }^{20}$ há grande incidência de caninos com suas raízes sobre os incisivos laterais, no entanto observamos também muitos caninos próximos à linha média. Quanto à disposição vertical dos caninos, este estudo observou grande porcentagem dedentes localizados no terço médio da raiz dos incisivos ${ }^{19}$. Resultados semelhantes foram obtidos por Power et $\mathrm{al}^{21}$. Reunindo os resultados obtidos, verificou-se que a maior parte dos caninos não irrompidos localiza-se próxima ao ponto de contato dos incisivos central e lateral estando sobreposto ao incisivo lateral, além disso, com estabelecimento e a padronização de métodos de localização de caninos não irrompidos obtém-se um melhor plano de tratamento.

Ao reabilitar um canino incluso é necessário todo cuidado no planejamento cirúrgico e ortodôntico antes da realização do tracionamento, para que não ocorram danos periodontais ou ainda que estes sejam minimizados ${ }^{22}$.

Em um estudo de caso clínico, realizado por Araújo et $\mathrm{al}^{23}$., foi abordado o uso da corrente de ouro para o tracionamento de caninos inclusos. Neste estudo foi 
utilizada corrente de ouro para a realização de tracionamento de dois caninos inclusos. Como vantagem desta técnica com este tipo de dispositivo ressaltou-se a possibilidade de acompanhar a evolução do tratamento pela quantidade de elos visualizados radiograficamente, além de sua biocompatibilidade. O paciente C.S.V., de 16 anos, do gênero feminino, foi encaminhado para tratamento ortodôntico. Ao exame clínico não se observou abaulamento das corticais dos caninos superiores, e os dentes 53 e 63 estavam presentes na arcada. Para complementar o diagnóstico foi realizado exame radiográfico utilizando a técnica de Clark, identificando os caninos superiores posicionados por palatino em relação aos dentes adjacentes. $\mathrm{O}$ tratamento foi dividido em duas cirurgias para exposição do dente e colagem do acessório, uma de cada lado. Foi feita a exodontia do dente 53 e o dente 23 foi identificado mais facilmente por palatino, por estar em posição mais favorável. Como método preventivo, o fio de sutura foi passado em um elo da corrente, presa por uma pinça hemostática curva, evitando possível queda para a orofaringe da paciente. 30 dias após já havia uma cicatrização sem sinais de inflamação ao redor do canino direito. Um mês após a realização da primeira cirurgia foi realizada a segunda. O pós-operatório do lado esquerdo foi moderado, com leve edema e dor controlados com analgésico. 45 dias após, observou-se um ótimo reparo e cicatrização da área, sem sinais de inflamação. Após as fases de exposição cirúrgica, os caninos permanentes foram submetidos ao tracionamento ortodôntico e posicionados na arcada dentária. O método de tracionamento ortodôntico cirúrgico associado à colagem de acessórios de ouro é uma oportunidade real para tratamento de dentes inclusos.

A literatura relata essencialmente três técnicas cirúrgicas para tracionamento dos caninos inclusos, técnica de laçamento do fio ortodôntico ao redor da junção cemento-esmalte, perfuração da coroa ou colagem de dispositivo ortodôntico, sendo essa ultima a mais utilizada por ser mais conservadora, minimizando possíveis retrações ${ }^{24}$. Além disso, Landim et al. ${ }^{25}$ avaliaram que o prognóstico da intervenção ortodôntica em casos de caninos impactados depende de muitos fatores, sobretudo da posição, da angulação do canino na maxila e da possibilidade de haver anquilose. Quanto mais precoce a intervenção, melhor o prognóstico do tratamento e para as etapas cirúrgica e ortodôntica da técnica de tracionamento, deve-se basear na extensão do deslocamento e no trauma cirúrgico causado pela exposição da coroa ${ }^{25}$.

Capellozza et $\mathrm{al}^{26}$ realizaram um estudo sobre protocolos para a perfuração do esmalte para o tracionamento de caninos (PETC), especificamente os caninos superiores, mais acometidos pelas anomalias de posicionamento, também denominadas disgenesias. Foram abordadas as vantagens e desvantagens da petc em relação à colagem de acessório para o tracionamento de caninos (CATC), evidenciando casos clínicos de vários níveis de complexidade, tendo como ponto de partida a literatura e a experiência clinica com elevado índice de sucesso ${ }^{26}$.

De acordo com esses autores na técnica petc há menor tempo cirúrgico, menor risco de um novo procedimento cirúrgico, possibilidade de aplicação de força no longo eixo do dente com magnitude melhor estabelecida e menor manipulação dos tecidos, inclusive o folículo pericoronário. O folículo pericoronário $(\mathrm{FP})$ é de extrema importância na erupção do elemento dentário, uma vez que libera mediadores, juntamente com outros ativados a partir de sua ação induz a reabsorção óssea, fenômeno essencial na erupção dentária ${ }^{27,28}$

A colagem de acessório para o tracionamento de caninos (catc) tem como vantagens menor custo biológico, por preservar o dente não perfurando o esmalte dentário e consequentemente menor risco de danos pulpares, em contrapartida existe maior manipulação do tecido pericoronário (folículo pericoronário), risco de novo procedimento cirúrgico e pouco controle de magnitude de forças $^{27}$.

De acordo com estudos fatores como aplicar excessiva ou extensivamente ácidos e outros produtos para facilitar a colagem dos dispositivos necessários para a fixação dos fios de tracionamento, remover todo o folículo pericoronário, abrir grandes janelas para expor o esmalte, assim como o envolvimento do colo dentário com fio metálico para tracionamento podem levar a reabsorção cervical externa, comprometendo o canino tracionado ortodonticamente $^{29}$.

Para execução do planejamento do tracionamento ortodôntico é necessário considerar a delicada estrutura da junção amelocementária, evitar manipulação cirúrgica desnecessária da região cervical, e não escoar produtos químicos, como ácidos. Assim à medida que o dente movimenta-se em direção oclusal, os tecidos pericoronários não são dilacerados ou "rasgados". A remodelação tecidual normal atende à demanda funcional e vai se adequando a esse movimento dentário de extrusão ${ }^{29}$.

De acordo com o estudo de Consolaro ${ }^{30}$ os diversos obstáculos que podem impedir a irrupção do canino. Muitas vezes o canino não irrompe, pois não ha espaço suficiente para alinhar-se. Assim o canino pode irromper parcialmente ou ficar mal posicionado, localizar-se na vestibular ou no palato, ou ficar alojado intraósseo. Existem casos em que há espaço mésio-distal no arco e ainda assim o canino não irrompe, esse espaço pode ser natural ou obtido ortodonticamente, isso porque o que determina a erupção do canino é a presença do folículo pericoronário. Esse folículo pericoronário ou saco pericoronário é unido à coroa através do epitélio reduzido, que um dia formou o esmalte. No seu tecido conjuntivo ainda temos muitos cordões e ilhotas epiteliais da lamina dentaria. As células do folículo pericoronário principalmente as células epiteliais liberam mediadores químico para as células ósseas vizinhas. Dentre esses fatores o EGF que estimula a reabsorção óssea periconária permitindo assim o dente irromper na arcada dentaria. Se o espaço na boca for menor que 1,5 do tamanho mesiodistal do canino não haverá espaço para o folículo pericoronário, e assim o canino não sofrerá erupção ${ }^{30}$.

Ferrarazo et al. $^{31}$ avaliaram sobre o assunto e verificaram que o processo de desenvolvimento do canino é o mais complexo e demorado e que diversos fatores podem interferir na irrupção do canino. O diagnóstico precoce é fundamental para o correto desenvolvimento da oclusão e para um tratamento eficaz e menos prolongado. A exodontia do canino decíduo, obtenção de espaços no arco dentário e o monitoramento radiográfico são protocolos atuais para o tratamento de certos casos, a fim de evitar ou minimizar terapias ortodônticas complexas e intervenções cirúrgicas.

No estudo de Zasciurinskiene et al. ${ }^{32}$ foi investigado o impacto do tratamento cirúrgico ortodôntico e a posição vertical e horizontal inicial sobre a saúde periodontal dos caninos superiores impactados na face palatina e dentes adjacentes. A amostra deste estudo retrospectivo incluiu 32 pacientes, sendo 22 mulheres e 10 homens, com caninos superiores unilaterais impactados por palatino previamente tratados. A duração do tratamento foi de $17,1 \pm 6,7$ meses 
(intervalo: 6-30 meses). A idade média dos pacientes para o exame periodontal foi de 18,2 $\pm 5,1$ anos (intervalo: 12-42 anos), três meses após a remoção dos aparelhos fixos. A posição inicial do canino impactado foi avaliada em relação ao incisivo lateral adjacente através de imagem panorâmica. Todos os pacientes foram tratados durante um período de quatro anos e foram submetidos à cirurgia com a técnica de erupção fechada. Um fio ortodôntico ligado a uma corrente foi instalado na superfície do canino impactado após exposição cirúrgica e o retalho foi reposicionado, sendo que a corrente se estendeu através do retalho. $\mathrm{O}$ aparelho fixo foi utilizado posteriormente para o posicionamento final do canino na arcada dentária. A avaliação periodontal póstratamento foi realizada por um periodontista 3 meses após a remoção dos aparelhos fixos e instalação de aparelho de contenção removível. Foram avaliadas profundidade de bolsa periodontal e recessão gengival no primeiro prémolar, canino e incisivo lateral. Para a detecção das áreas mais profundas foi utilizada sonda periodontal inserida paralelamente ao longo eixo vertical do dente e em torno das superfícies dentárias mesiovestibular (ML), vestibular (L), distovestibular (DL), distopalatina (DP), palatina (P), e mesiopalatina (MP). Técnica semelhante foi utilizada para avaliar a recessão gengival, porém medindo a distância da junção amelocementária até a margem gengival nas superfícies dentárias mesial (M), palatina (P), distal (D) e vestibular (L). Os autores observaram um aumento significativo na profundidade de bolsa na face mesiopalatina (MP) do canino que passou por tratamento cirúrgicoortodôntico em torno de $3,1 \mathrm{~mm}( \pm 1,0 \mathrm{~mm})$. A profundidade da bolsa periodontal se mostrou maior no grupo de caninos impactados com posição vertical inicial V2 $(2,93 \pm 0,92 \mathrm{~mm})$ do que no grupo com posição vertical V1 $(2,33 \pm 1,14 \mathrm{~mm})$. Para os autores, o tratamento cirúrgico-ortodôntico de caninos superiores impactados oferece uma condição periodontal mais ou menos aceitável, dependendo da posição inicial vertical e horizontal do dente.

\section{DISCUSSÃO}

Quando consideradas a etiologia e incidência, estudos de Jarjoura et al. ${ }^{33}$, relataram alta incidência de impactações do canino por palatino associadas com ausência do incisivo lateral ou de tamanho diminuído. Entretanto, também é possível que a posição ectópica do incisivo lateral seja um bloqueio para irrupção do canino, fato esse que pode explicar tamanha porcentagem de laterais com reabsorção. Jacoby ${ }^{34}$ concluiu que $85 \%$ dos casos de caninos superiores retidos por palatino têm espaço suficiente no arco e argumentou que uma deficiência no comprimento do arco levará somente o canino a erupcionar por vestibular.

O paciente e/ou responsáveis devem estar cientes das vantagens e riscos do tratamento já que as impactações dentárias podem causar vários problemas como reabsorção das raízes dos dentes vizinhos, perda do comprimento do arco, formação de cisto dentígero, infecções locais, dor reflexa entre outros ${ }^{35}$. Os caninos superiores permanentes têm a maior incidência de impactação depois dos terceiros molares, manifestando-se estatisticamente em $2 \%$ da população ${ }^{36}$.

Estudos voltados para o diagnóstico, tais como os de Manzi et al. ${ }^{28}$ a anamnese, o exame clínico e radiográfico são utilizados para o diagnóstico de caninos inclusos. É frequente a associação de métodos por imagem convencionais para o diagnóstico, e a associação da tomografia computadorizada é de fundamental importância para se analisar com precisão a exata localização, posição, e a relação com estruturas e dentes adjacentes, para que assim seja instituído um tratamento adequado para o caso.

Consolaro $^{30}$ verificou que o uso da tomografia e das imagens 3D antes de iniciar o tracionamento ortodôntico pode contribuir no planejamento, mas também elimina a possibilidade da preexistência de processos como a própria Reabsorção Cervical Externa, a anquilose alveolodentária e a reabsorção dentária por substituição nos dentes a serem tracionados.

Dados da literatura abordando o tratamento de caninos inclusos permanentes orientam o ortodontista a considerar as várias opções de tratamento disponível, incluindo os seguintes: aguardar a irrupção espontânea do canino retido, assim tem sido descritos na literatura a retirada de interferências mecânicas como extranumerários, patologias e até recuperação de espaço ${ }^{37}$. Se o paciente assim preferir; é importante que se faça um controle radiográfico periódico para avaliar alterações patológicas ${ }^{38}$.

No estudo de Bishara $^{39}$ para extração do canino retido devem ser considerados os seguintes aspectos: a anquilose, reabsorção interna ou externa, raiz dilacerada, retenção severa, quando houver formações patológicas ou quando o indivíduo não desejar tratamento cirúrgico ortodôntico.

A exposição cirúrgica e posterior tratamento ortodôntico é o caminho mais utilizado. Deve ser muito bem planejado com o Cirurgião para a obtenção de melhores aspectos estéticos e periodontais ao final do tracionamento ${ }^{40}$.

De acordo com McBride ${ }^{40}$ para prognóstico favorável nos tracionamentos ortodônticos alguns fatores devem ser considerados: a idade do indivíduo, as condições de espaço, o posicionamento sagital e transversal do canino retido (coroa e raiz) todos esses são de grande importância para o prognóstico do caso. Quando os caninos estão localizados por vestibular, duas técnicas cirúrgicas podem ser empregadas. A exposição radical por meio da retirada da mucosa alveolar, osso alveolar e folículo dentário, o que pode causar problemas periodontais, devido à anatomia da mucosa vestibular ${ }^{41}$, podendo o canino ser tracionado por meio de dispositivo colado a superfície do dente ou então por perfuração do esmalte dentário como descrito por Capellozza ${ }^{26}$.

O cantilever é uma das opções de tracionamento para o canino impactado por palatino, sendo que nessa técnica existe a possibilidade de aplicar os princípios biomecânicos, controlando melhor os efeitos colaterais gerados pelos aparelhos ortodônticos, respeitando a condição periodontal com forcas leves e criando sistema de forças individuais para cada caso ${ }^{41}$

\section{CONCLUSÃO}

Em se tratando de caninos impactados é necessário o exato diagnóstico, para isso o profissional deve lançar mão de exames complementares, bidimensionais representados pelas radiografias de diversas normas (frontal, periapical, panorâmica) e tridimensional sendo a tomografia computadorizada com o melhor detalhamento de estruturas adjacentes ao dente impactado.

De acordo com os dados analisados na literatura quanto mais precocemente diagnosticados os caninos permanentes impactados melhor serão o prognóstico e tratamento do caso. Com base nos estudos literários a terapêutica mais realizada para tratamento dos caninos é o tracionamento realizado através da exposição cirúrgica, e 
posterior colagem de dispositivo ortodôntico. Vale ressaltar que $\mathrm{o}$ folículo pericoronário (FP) deve sempre ser preservado nesse processo, uma vez que sua remoção em excesso ou mesmo exposição do FP a ácidos podem comprometer a irrupção do dente a ser tracionado. A técnica de perfuração do esmalte dentário também é uma opção para tracionamento, porém exige muito domínio da técnica pelo profissional, uma vez que um erro na perfuração pode comprometer a vitalidade pulpar do elemento a ser tracionado.

\section{REFERÊNCIAS}

1. Al-nimri K., Gharaibeh T. Space conditions and dental and occlusal features in patients with palatally impacted maxillary canines: an etiological study. Eur J Orthod. 2005; 27(5):461-5.

2. Ericson S, Kurol J. Resorption of incisors after ectopic eruption of maxillary canines. A CT study. Angle Orthod. 2000; 70(6):415-23.

3. Brito AM, Fraga CFF, Goursand D, Costa EN, Grossi E, Júnior JFR. Impactação de caninos superiores e suas consequências: relato de caso clínico. J Bras Orthod Ortop Facial. 2003; 48(4):453-9.

4. Cappellette M, Júnior MC, Fernandes LCM, Oliveira AP, Yamamoto LH, Shido FT et al. Caninos permanentes retidos por palatino: diagnóstico e terapêutica - uma sugestão técnica de tratamento. Rev Dent Press Ortodon Ortop Facial. 2008; 13(1):60-73.

5. Msagati F, Simon ENM, Owibingire S. Pattern of occurrence and treatment of impacted teeth at the Muhimbili National Hospital. BMC Oral Health. 2013;13(37):1-6.

6. Peck S, Peck L, Kataja M. The palatally displaced canine as a dental anomaly of genetic origin. Angle Orthod. 1994; 64(4):249-56.

7. Garib DG, Henriques JFC, Freitas MR, Janson GRP. Caninos superiores retidos: Preceitos clínicos e radiográficos. Rev Dental Press Ortodon Ortop. 1999; 4(4):14-20.

8. Aizenbud, D. Bilateral maxillary impacted canines: a challenge for the lingual orthodontic technique Lingual News. 2003; 1(2):5.

9. Maahs M, Berthold T. Etiologia, diagnóstico e tratamento de caninos superiores permanentes impactados. Rev Cienc Med Biol. 2004; 3(1):130-8.

10. Fox NA, Fletchr GA, Horner K. Localising maxillary canines using dental panoramic tomography. Br Dent $\mathbf{J}$. 1995; 179(11/12):416-20.

11. Franco AA, Paixão GB, Cevidanes LHS, Chaves Júnior CM. Abordagem multidisciplinar dos caninos superiores permanentes impactados. Ortodontia; 39(4):350-9.

12. Quirynen M, Heij DGO, Adriansens A, Opdebeeck HM, Steenberghe D. Periodontal health of orthodontically extruded impacted teeth: a split-mouth longterm clinical evaluation. J. Periodontol. 2000; 71(11):1708-14.

13. Brown NL, Sandy JR. Spontaneous improvement in position of canines from pparently hopeless positions. Int J Paediatr Dent. 2001; 11(1):64-8.

14. Iramaneerat, S.; Cunningham, S. J.; Horrocks, E. N. The effect of two alternative methods of canine exposure upon subsequent duration of orthodontic treatment. Int $\mathbf{J}$ Paediatr Dent. 1998; 8(2):123-9.

15. Mason C, Papadokou P, Roberts GJ. The radiographic localization of impacted maxillary canines: a comparison of methods. Eur J Orthod. 2001; 23(1):2534.

16. Alves EP, Montagner AF Antoniazzi SP, Oliveira LFD. Prevalência e posição de caninos superiores impactados e sua relação com reabsorção radicular. RFO Passo Fundo. 2014; 19(2):180-4.

17. Gavel V, Dermaut L. The effect of changes in tooth position of unerupted canines on cephalograms. Eur $\mathbf{J}$ Orthod. 2003; 25(1):49-56.

18. Manzi FR, Ferreira EF, Rosa TZS, Valerio CS. Uso da Tomografia Computadorizada para Diagnóstico de Caninos Inclusos. Rev Odontol Bras Central. 2001; 20(53):103-7.

19. Martins PP, Gurgel JA, Sant'ana E, Ferreira Júnior O, Henriques JFC. Avaliação radiográfica da localização de caninos superiores não irrompidos. Rev Dental Press Orthod Ortop Facial. 2005; 10(4):106-14.

20. Ericson S, Kurol J. Early treatment of erupting maxillary canines by extraction of the primary canines. Eur J Orthod. 1988; 10(4):283-95.

21. Power SM, Short MB. An investigation into the response of palatally displaced canines to the removal of deciduous canines and an assessment of factors contributing to favourable eruption. Br J Orthod. 1993; 20(3):215-23.

22. Tanaka OM, Guidelli S, Ribeiro JS, Guariza Filho O, Taffarel IP. Os desafios biomecânicos na movimentação de caninos inclusos em adultos. Rev Clin Ortodon Dental Press. 2008; 7(1):90-7.

23. Araújo MM, Teixeira TM, Araújo AM, Kimaid A. Tracionamento de canino incluso com a utilização da corrente de ouro como acessório ortodôntico: relato de caso clínico. Rev Clin Ortodon Dental Press. 2006; 5(2):52-8.

24. Callá L, Cuffari L. O que o ortodontista precisa saber para indicação de procedimentos cirúrgicos ortodônticos em caninos retidos. JBO - J Bras Ortodon Ortop Facial. 2004; 9(53):466-73.

25. Landim FS, Freitas GB, Rocha NS, Caubi AF, Holanda RJ. Avaliação clínicoradiográfica dos caninos após tratamento orto-cirúrgicos. Rev Cir Traumatol BucoMaxilo-Facial Camaragibe. 2010; 10(4):103-10.

26. Capellozza Filho L, Consolaro A, Cardoso MD, Siqueira M. Perfuração do esmalte para o tracionamento de caninos: vantagens, desvantagens, descrição da técnica cirúrgica e biomecânica. Dental Press J Orthod; 16(5):172-205.

27. Consolaro A. Tracionamento dentário: mitos, coincidências e fatos - Parte II. Este procedimento provoca anquilose alveolodentária? Rev Clín Ortod Dental Press. 2003; 2(6):100.

28. Consolaro A. O tracionamento ortodôntico representa um movimento dentário induzido! Os quatro pontos cardeais da prevenção de problemas durante $\mathrm{o}$ tracionamento ortodôntico. Rev Clín Ortod Dental Press. 2010; 9(4):105-10.

29. Consolaro A. Tracionamento ortodôntico: possíveis consequências nos caninos superiores e dentes adjacentes Parte 2: reabsorção cervical externa nos caninos tracionados. Dental Press J Orthod. 2010; 15(5):23-30.

30. Consolaro A. Porque o canino não vem? In: Consolaro A, Consolaro MFMO (eds.). Controvérsias na ortodontia e atlas de biologia da movimentação dentária, Maringá: Dental Press, 2008

31. Ferrazzo VA, Domingues GC, Santos JR JH, Vargas 
DA, Ferrazzo KL. Caninos superiores impactados: revisão de literatura e relato de caso clínico. Ortodontia SPO. 2005; 3(38):247-54.

32. Zasciurinskiene E, Bjerklin K, Smailiene D, Sidlauskas A, Puisys A. Initial vertical and horizontal position of palatally impacted maxillary canine and effect on periodontal status following surgical-orthodontic treatment. Angle Orthodontist. 2008; 78(2):275-80.

33. Jarjoura K, Crespo P, Fine JB. Maxillary canine impactions: orthodontic and surgical management. Compend Contin Educ Dent. 2002; 23(1):23-31.

34. Jacoby $\mathrm{H}$. The etiology of maxillary canine impactions. Am J Orthod. 1983; 84(2):125-32.

35. Quirynen M, Heij DGO, Adriansens A, Opdebeeck HM, Steenberghe D. Periodontal health of orthodontically extruded impacted teeth: a split-mouth longterm clinical evaluation. J. Periodontol. 2000; 71(11):1708-14

36. Stewart JA, Heo G, Glover KE, Williamson PC, Lam EWN, Major PW. Factors that relate the treatment duration for patients with palatally impacted maxillary canines. Am J Orthod Dentofacial Orthop. 2001; 119(3):216-25.

37. Duncan WR, Ashrafi MH. Management of nonerupted maxillary anterior tooth. J Am Dent Assoc. 1983; 106(5):640-4.

38. Tormena Júnior R, Vedovello Filho M, Ramalho S, Wassall A, Valdrighi T, Cristina H. Caninos superiores retidos: uma reabilitação estética e funcional. J Bras Ortodon Ortop Facial. 2004; 9(49):77-86.

39. Bishara SE. Impacted maxillary canines: a review. Am J Orthod Dentofacial Ortothop. 1992; 101(2):159-71.

40. McBride LJ. Traction - a surgical orthodontic procedure. Am J Orthod. 1979; 76(3):287-99.

41. Bastos MO. Cantilever para tracionamento de caninos inclusos palatinamente. Rev Clin Ortodon Dental Press. 2003; 2(1):5-17.

\section{CONFLITO DE INTERESSES}

Os autores declaram não haver conflitos de interesse.

\section{AUTOR PARA CORRESPONDÊNCIA}

André Pìnheiro de Magalhães Bertoz

andrebertoz@foa.unesp.br

Submetido em 11/09/2017

Aceito em 18/10/2017 